Background Viral bronchiolitis is a common lower respiratory infection during infancy. 50\% of infants with acute Bronchiolitis develop Post bronchiolitis wheezing (PBW) that may cause childhood asthma. Vitamin A deficiency impairs epithelial integrity, systemic immunity and increases the severity of childhood respiratory infections such as RSV. In this study the effect of vitamin A in improving PBW has been evaluated.

Methods This was a double blind pilot trial on 84 patients aged 2 to 12 months old who were admitted in Emam Sajad Hospital in Yasuj (Iran) from October 2012 to October 2013. The patients were diagnosed by history, physical examination and chest $\mathrm{x}$-ray by a paediatrician. The 84 individuals were put in case and control groups (42 in each group). The case group received $5000 \mathrm{IU} / \mathrm{kg}$ Vitamine A intramascularly, plus cold and wet oxygen, and beta 2 agonist (ventolin) by nebulizer, and the control group received the same except vitamin A.1,2 and 3 weeks after treatment the patients were examined by the same paediatrician and were compared based on severity of wheezing, and response to the treatment (decrease in wheezing, distress and cough).

Findings The diferences of demographic data and severity of wheezing were not significant between the groups before treatment. There were significant differences in response to treatment between two study groups after 1, 2 and 3 weeks following treatment $(\mathrm{p}=0.045, \mathrm{p}=0.001$ and $\mathrm{p}=0.001$ respectively). No side effects were seen during and after the course of treatment.

Conclusion Usual treatments together with Vitamin-e A have stronger effect on reducing post bronchiolitis wheezing, compared with usual treatments alone.

\section{PO-1019 CLINICAL CHARACTERISTICS AND RISK FACTORS OF SEVERE RESPIRATORY SYNCYTIAL VIRUS-ASSOCIATED ACUTE LOWER RESPIRATORY TRACT INFECTIONS IN HOSPITALISED INFANTS}

L Liu, X Zhang, L Qian, L Wang, Y Yin. Department of Respiratory Medicine, Children's Hospital of Fudan University, Shanghai, China

\subsection{6/archdischild-2014-307384.1635}

Objective To investigate the clinical characteristics and analyse risk factors for severe respiratory syncytial virus (RSV) infection in hospitalised infants with acute lower respiratory tract infections (ALRIs).

Methods A retrospective review of the medical records of infants with RSV-associated ALRIs between March 1st, 2011 and February 29th, 2012 was done.

Results Among 913 RSV-associated ALRI infants, there were 288 severe infants (31.5\%). The severe cases accounted for $4.2 \%$ of the hospitalised children and gave a hospital RSV mortality rate of $1.0 \%$. The occurrence of severe RSV infection had a seasonal variation, with a peak in winter (45.6\%). The proportions of cases with tachypnea, apnea, cyanosis, and fine rales were significantly higher in the severe ALRIs group (all p < 0.001). The incidences of bronchitis, pneumonia and readmission in the severe group were higher than those in the nonsevere infants during the one-year follow-up (all $\mathrm{p}<0.001$ ). Multivariate logistic regression determined the factors increasing the risk of severe RSV infection were: low-birth-weight [1.698 (1.028 2.805)], under 3 months of age [3.385 (2.174 5.271)], congenital heart disease [1.667 (1.149 2.418)], bronchopulmonary dysplasia [8.505 $(1.731 \sim 41.780)]$, and airway abnormalities [2.246 (1.008 5.005)].
Conclusions Severe RSV infection is associated with a high hospitalisation and mortality. Preventative measures should be targeted against high-risk hospitalised infants, especially those who are low-birth-weight, under 3 months of age, and with underlying diseases.

Keywords Respiratory syncytial virus, Acute lower respiratory tract infections, Risk factor

\section{PO-1020 VALIDATION OF A NUTRITIONAL SCREENING TOOL FOR PATIENTS WITH CYSTIC FIBROSIS}

${ }^{1}$ Miss Simon, ${ }^{1} \mathrm{~T}$ Maraschin, ${ }^{2} \mathrm{GC}$ Forte, ${ }^{2} \mathrm{JS}$ Pereira, ${ }^{3} \mathrm{EFA}$ Procianoy, ${ }^{4} \mathrm{M}$ Drehmer. ${ }^{1}$ Centro de Estudos em Alimentação e Nutrição, Hospital de Clínicas de Porto Alegre, Porto Alegre, Brazil; ${ }^{2}$ Nutrition, Hospital de Clínicas de Porto Alegre, Porto Alegre, Brazil; ${ }^{3}$ Pediatric Pulmonology Unit, Hospital de Clínicas de Porto Alegre, Porto Alegre, Brazil; 4 UFRGS, Hospital de Clínicas de Porto Alegre, Porto Alegre, Brazil

\subsection{6/archdischild-2014-307384.1636}

Background The study aim was to validate a specific nutritional risk screening tool for patients with cystic fibrosis (CF) based on clinical variables, anthropometric parameters, and dietary intake. Methods Cross-sectional study compared present nutritional screening tool and two established nutritional assessment instruments. The kappa coefficient was used for between-group comparisons, and the sensitivity and specificity of the new instrument for diagnosis of nutritional risk were calculated.

Results 82 patients (51\% female, mean age 6-18 years) were enrolled in the study. There was good agreement between the proposed screening tool and the McDonald tool $(\mathrm{k}=0.804$; $95 \% \mathrm{CI}, \mathrm{p}<0.001)$ and a sensitivity and specificity of $85 \%$ and 95\% respectively. Agreement with the CFF criteria was lower $(\mathrm{k}=0.418, \mathrm{p}<0.001)$, and the sensitivity and specificity were both $72 \%$.

Conclusion The proposed tool exhibited satisfactory agreement with the comparator instruments, and can be recommended for early identification of failure nutritional in patients with CF.

Keywords Cystic fibrosis, nutritional assessment, nutritional status

\section{PO-1021 PHENOTYPIC EXPRESSION OF BRONCHIAL ASTHMA IN INDIAN CHILDREN: PRELIMINARY RESULTS FROM THE CHILDHOOD ASTHMA PHENOTYPE (CAP) COHORT}

JL Mathew, S Paikray, M Singh. Advanced Pediatrics Centre, Post Graduate Institute of Medical Education and Research, Chandigarh, India

\subsection{6/archdischild-2014-307384.1637}

Background Clinical experience suggests that childhood asthma phenotype in developing countries may be different from developed countries where majority of asthma is atopic.

Objective To study the phenotypic expression of bronchial asthma in Indian children with special reference to symptoms, features of atopy, family history, and exacerbating/relieving factors.

Methods The study was conducted with Ethics clearance and children were enrolled with written informed parental consent. Childhood asthma phenotype (CAP) cohort comprises 600 consecutively enrolled children $(<12 \mathrm{y})$ with asthma defined by NAEPR-III Guidelines. Enrolled children underwent a standard questionnaire to elicit demographic features, presenting symptoms, atopic features, and parental perception of exacerbating/ relieving factors. Older children $(>8 y)$ underwent spirometry. 